\title{
Intelligent Modularized Reconfigurable Mechanisms for Robots: Development and Experiment
}

\author{
Wenfu Xu ${ }^{1}{ }^{0}$, Liang Han ${ }^{1}$, Xin Wang ${ }^{1}$, Han Yuan $^{1 *}$ and Bin Liang ${ }^{2}$
}

\begin{abstract}
With the development of intelligent flexible manufacturing, traditional industrial manipulators with a single configuration are difficult to meet a variety of tasks. Reconfigurable robots have developed rapidly which could change their configurations and end effectors for different tasks. The reconfigurable connecting mechanism (RCM) is a core component of reconfigurable robots. In this paper, two types of intelligent modularized RCMs with light weight, high payload, and large pose (position and attitude) error tolerance are developed. One is driven by shape memory alloy (SMA) and recovery spring. It is locked by steel balls and key. The other is driven by electromagnetic coil and locked by permanent magnet and key. The locking principle, mechanical system and control system of the two RCMs are detailed introduced. Both of them meet the requirements of high precision and high payload in the industrial field. Finally, the developed RCMs are respectively integrated to a practical robot and experimented. The experiment results verified the performance of the two RCMs.
\end{abstract}

Keywords: Reconfigurable connecting mechanisms, Shape memory alloy, Electromagnetic, Reconfigurable manipulator

\section{Introduction}

Intelligent and flexible manufacturing requires that industrial manipulators can be quickly deployed and applied to different scenarios [1]. The manipulator with a single configuration and a single end effector cannot meet the complex and variable tasks. Reconfigurable manipulators have been used in some applications like space field, industrial field, and environmental detection field because they could change their configurations and end effectors for different tasks. The reconfigurable manipulator has multiple advantages like improving the environmental adaptability, maintenance capability, and reducing cost.

The currently developed reconfigurable robots can be divided into two types including reconfigurable

\footnotetext{
${ }^{*}$ Correspondence: yuanhan@hit.edu.cn

${ }^{1}$ School of Mechanical Engineering and Automation, Harbin Institute of Technology, Shenzhen 518055, China

Full list of author information is available at the end of the article
}

manipulator and self-reconfigurable cell robot. Selfreconfigurable cell robots is composed of a series of independent electromechanical modules. Each module has the function of connecting, disconnecting and crossing adjacent modules autonomously. These modules can be connected into humanoid robots, quadruped robots, wheeled robots, etc. It is generally used for environmental detection. The RCMs of self-reconfigurable cell robots do not require large load capacities. Reconfigurable manipulators is composed of joint modules and link modules. The joint modules and the link modules can be connected to form a robotic arm. It is generally used for industrial field. The RCMs of reconfigurable manipulators need large load capacities. In industrial field, many reconfigurable manipulators have been developed. RMMS [2] developed by Carnegie Mellon University is the first reconfigurable robot. RMMS expands the concept of the modular robot. It not only realizes the reconfigurable mechanical structure, but also the reconfigurable electronic, hardware and control algorithms 
RMMS adopts the distributed control system. Each module contains sensors, motors, brakes, actuators, communication bus, etc. The modules are connected by simple rotation and locking by the quick-connect mechanical interface. In Ref. [3], the locking mechanisms in robotics are reviewed. The locking mechanisms are used to connect modules, reconfigure robots, lock joints, etc. Based on different locking principles, the locking mechanisms are divided into three types including mechanical locking, friction-based locking, and singularity locking. Each type is discussed in the paper. In Ref. [4], an approach for reconfiguring industrial robots using several modules is proposed. These modules include joint module, link module, and end-effector module. Comparing to the existing commercial robots, the reconfigurable industrial robot can reduce the cost. MoRSE [5] developed by AMTEC company has three basic modules including rotating joint modules, prismatic joint modules, and link modules. Based on the MoRSE, a variety of reconfigurable modular robot systems are built by Nanyang Technological University. Kinematics, dynamics, calibration, and configuration have also been studied [6]. In Ref. [7], an under actuated self-reconfigurable robot was presented. It includes the active modules and passive modules. The passive modules can connect with the active modules with the docking system to expand the link length. The docking system uses the key and spring to lock. When the passive module is pushed and rotated, it will be pulled to the position between two teeth by the spring, and the two modules are locked. However, this docking system may shake due to deformation of the spring. In Ref. [8], considering the payload to weight ratio and limited manipulation ability problems in reconfigurable robots, a new joint module with the assisted spring is developed. The spring is assembled between the brake and the motor shaft. When the reconfigurable robot adds the modules and expands the workspace, the payload problem can be solved to some extent. TOMMS system developed by TOSHIBA contains joint modules, and link modules [9]. Through mechanical and electrical connection, a variety of manipulators can be easily assembled. The similar systems also include LWR-III developed by German Aerospace Center [10], the Power Cube module by Schunk Company [11]. However, these modular reconfigurable manipulators cannot complete the reconstruction autonomously. In Ref. [12], a new reconfigurable mechanism is developed by locking and unlocking the passive joints. The passive joints are equipped with the brake mechanism. It does not need the RCM to connect the modules. But the types of the transformable configurations are limited.

Self-reconfigurable cell robots have attracted a lot of researches. It can be divided into lattice type robot, chain type robot, mobile type robot, and hybrid type robot [13]. In Ref. [14], a novel reconfigurable continuous minimally actuated track robot (RCTR) is developed. The robot is actuated by three motors. Two motors are used to drive the track and the third motor is used to change the shape. Its shape can be changed to avoid different obstacles. It is actuated using a servo motor which moves the locking pin from the center to either side. Catoms [15] is a modular robot with a planar array structure from Carnegie Mellon University. The relative position is changed by the adsorption and repulsion of electromagnets between modules to achieve deformation. In Ref. [16], a modular self-reconfigurable robot UBot is developed. It contains active modules and the passive modules. Each active module has four hooks on the connecting surface. The hooks are driven by a motor to connect the passive module. In Ref. [17], the reconfigurable spherical robot is presented. It contains two hemispherical modules. It can transform from a spherical mode into a three-legged walking mode using a linear motor. The chain type robot forms a chain or tree structure like a manipulator or a bionic robot through the connection between multiple modules. PolyBot [18] includes active modules and node modules. The active module has two connectors. The node module is a rigid cube with six connectors on its six surfaces. By connecting multiple modules, it can be assembled into various configurations such as snakelike robot, multi-legged robot, and crawler robot. Based on the PolyBot platform, Yim et al. [19] carried out a lot of research on robot technologies including self-reconfiguration, robot gait, and space robot. In Ref. [20], the self-reconfigurable modular robot Roombot is developed. One module consists of 4 interconnected half-spheres that can continuously rotate around each other by 3 motor units. Each module has 10 connection plates. Each connection plate can connect with any other connection plate with the retractable hooks. Infrared sensors and hall sensors are used to check the two plates correctly oriented. CONRO [21] is a modular robot developed by the University of Southern California. Each module has two degrees of freedom including a modular body, an active connector and a passive connector. The connector is positioned through pin and hole and locked through the rotating hook driven by the SMA. Each module of the mobile type cell robot can move independently. Generally, the reconstruction of the structure and the motion control are realized through wireless communication. In Ref. [22], a reconfigurable robot called hTetro is developed for floor cleaning. It consists of four modules with the same size and the different function. The robot uses six motors for mobility and three motors for transformation. The motor rotates the module to change the configuration. In Ref. [23], referring to the origami robots, a 
new robotic platform called Mori is developed. The modules can connect each other and fold up to create a threedimensional reconfigurable robot from two-dimensional structure. SWARM-bot [24] is composed of multiple small robots called s-bots. Each s-bot is a highly autonomous mobile robot which can identify the surrounding environment and complete navigation, operation and other tasks independently. It is equipped with infrared sensor, acceleration sensor, temperature sensor, force sensor and etc. Multiple s-bot can autonomously connect with each other to form the SWARM-bot. SWARMbot can complete more complex tasks that a single s-bot cannot complete. In Ref. [25], the reconfigurable hybrid wheel-track mobile robot called RHMBot is developed. It can construct four modes like wheel mode, tracked mode, climbing and roll-over mode through deformable track belt. In Ref. [26], a highly reconfigurable sprawl tuned robot RSTAR is developed. It can extend the distance between its body and legs to avoid challenging obstacles, crawl over flexible and slippery surfaces and even climb vertically in a tube or between two walls. Hybrid type robots often have multiple features of the above types. M-TRAN [27] is a hybrid self-reconfigurable robot that has both lattice structure and chain structure. Each module consists of a connecting link and two semi-cylinders. The power supply and communication bus between the modules are connected via contacts. M-TRAN has a strong self-reconfiguration capability. M-TRAN II [28] and M-TRAN III [29] are upgraded versions of the M-TRAN robot. M-TRAN and M-TRAN II uses SMA and armature to connect modules. M-TRAN III uses rotating hook to connect modules. Superbot [30] designed by the University of Southern California is also a lattice/chain hybrid robot which has a flexible gait. HIT MSR I [31] and HIT MSR II [32] developed by Harbin Institute of Technology are self-reconfigurable modular robots. HIT MSR I uses SMA and spring to lock. HIT MSR II uses rotating hooks driven by a micro motor to lock. Self-reconfigurable cell robots have many types of RCMs. However, most of them have complex structures and low payloads. They cannot be directly used in industrial field. Nevertheless, the principles can be referred for designing new RCMs of reconfigurable manipulators. In addition, the quick tool changing device is widely used in machining. Quick tool changing devices generally use hydraulic pressure or pneumatic pressure to lock the tools. The system is complex and difficult to maintain. Quick coupling is widely used in fluid or gas pipeline. It uses steel balls to lock. It needs to manually move the position of the slider to lock and unlock the two parts.

Some RCMs use the extra motor to drive the locking mechanism. It will increase weight, cost and energy consumption. RCMs in self-reconfigurable cell robots are difficult to meet the application requirements of high payload in industrial field. Therefore, we designed two types of RCMs with light weight, high payload, high reliability and big tolerance for self-reconfigurable manipulators. The main contributions of the paper are as follows. 1) Two types of RCMs are developed based on SMA and the electromagnetic respectively. 2) Without using motors and its drive board, the structure is simpler and the cost is lower. The RCMs have high payload to weight ratio. 3) With big tolerance design, two RCMs can complete docking process in the case of errors in the robotic arm.

This paper is organized as follows. In Section 2, the demand analysis and application concept of RCMs are analyzed. In Section 3, the locking principle, mechanical system and control system of the RCM using SMA are introduced in detail. In Section 4, the locking principle, mechanical system and control system of the RCM using electromagnetic are introduced in detail. In Section 5, the prototypes of two RCMs are established. The performance is verified by a series of experiments. Finally, we give the conclusion in Section 6.

\section{Demand Analysis and Application Concept}

\subsection{Demand Analysis}

The RCM developed in this paper is used for the reconfigurable robot in industrial area. Considering that the robotic arm often performs object carrying tasks, the RCM should have high payload and low weight. Considering the pose errors of the robotic arm, the RCM should have big tolerance. The RCM generally contains the drive source, the locking mechanism, the tolerance mechanism, the electrical connector, and the control system. In order to realize the simple structure, low cost and energy consumption, the driving source do not use the motor its related driving board. The components, methods, and characters of two RCMs are shown in Figure 1.

1) The tolerance mechanism is used to ensure the success of the connection under the position and attitude

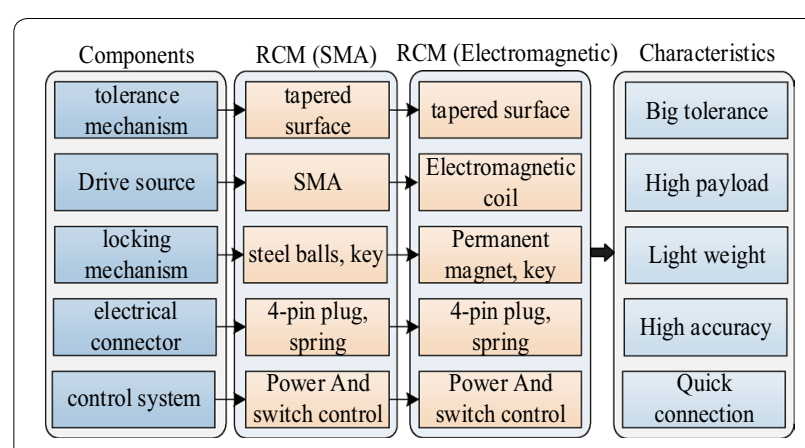

Figure 1 Components, methods, and characters of two RCMs 
errors. The tolerance mechanisms are designed both on the mechanical connecting part and electrical connecting part to improve the success rate of the connection and reduce the contact force on some parts. For mechanical part, the cone structure is often used as the tolerance. For electrical part, the spring is often used as the buffer mechanism. The electrical connection process starts when the active part and the passive part are successfully matched through the guiding of the taper. 2) The locking mechanism is used to lock the active part and the passive part of RCM after they complete the docking process. The freedom of the two parts is strictly limited. 3) The drive source refers to the device that triggers the locking mechanism to move. 4) The electrical connector is used to connect the power supply and communication wires. 5) The control system is used for power management and current (A) on and off.

The RCM used for the reconfigurable robot in industrial area should have light weight, high payload, big tolerance, and high accuracy. According to these requirements, two types of RCMs are developed. The first RCM uses SMA as the drive source and the steel balls to lock. The second RCM uses electromagnetic coil as the drive source and permanent magnet to lock. Both two RCMs have high load-to-weight ratio, small size, simple structure, and high reliability. They are suitable for industrial reconfigurable manipulators.

\subsection{Application Concept}

The reconfigurable manipulator may change its configuration and end effector for different tasks. Considering two typical working scenarios including changing the link to expand the manipulator's workspace and changing the tool to perform different operations, we demonstrate the processes of changing the link and tool as shown in Figure 2. First, the reconfigurable manipulator can be rapidly formed by a series of modules. Then, the manipulator can change its configuration and tool by connecting

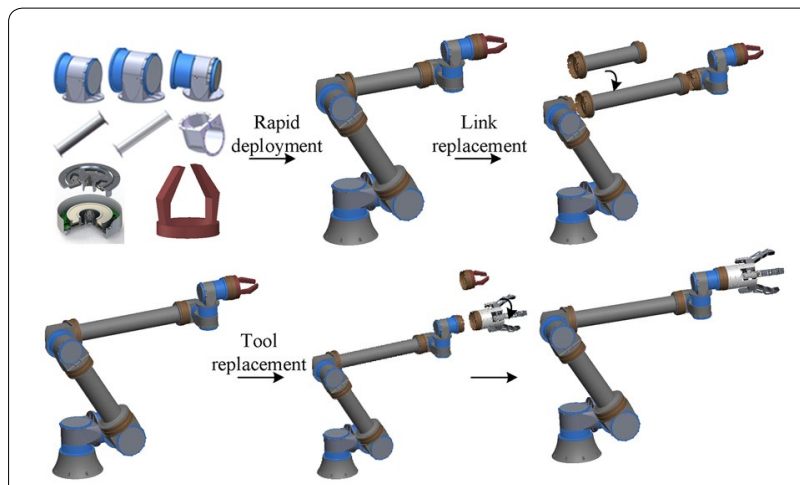

Figure 2 Process of changing the link and tool and disconnecting RCMs. Each module has an active part and a passive part of RCM. The robot can automatically complete these two processes.

\section{SMA-Driven Reconfigurable Mechanism}

\subsection{Structure Design}

\subsubsection{Overall Structure}

The overall structure of the RCM based on SMA is shown in Figure 3. It includes the active part and the passive part. The active part undertakes most of the connecting works. It mainly consists of the SMA and recovery spring as the driving source, the steel balls and key as the load-bearing parts, the slider as the part to control the position of the steel balls, the cone as the tolerance, the socket and buffer spring as the electrical connections, the insulation as the leakage prevention, and the controller board as the power management. The passive part cooperates with the active part, which is mainly composed of the plug, the cone, and the keyway. When the cones of the active part and the passive part are successfully fitted, the plug and the socket begin to connect to achieve electrical connection. Main material of the RCM is aluminum alloy. In order to reduce weight, the unbearable parts are removed.

\subsubsection{Working Principle}

Whether the SMA is powered determines the connection state of the RCM. When the SMA is powered on, its length is shortened. The recovery spring is compressed and the groove of the slider moves to opposite the hole position of the steel balls. The steel balls are dropped into the groove of the slider. The RCM works in the unlocked state. The passive part can be separated from the active part. The unlocked state is shown in Figure 4(a).

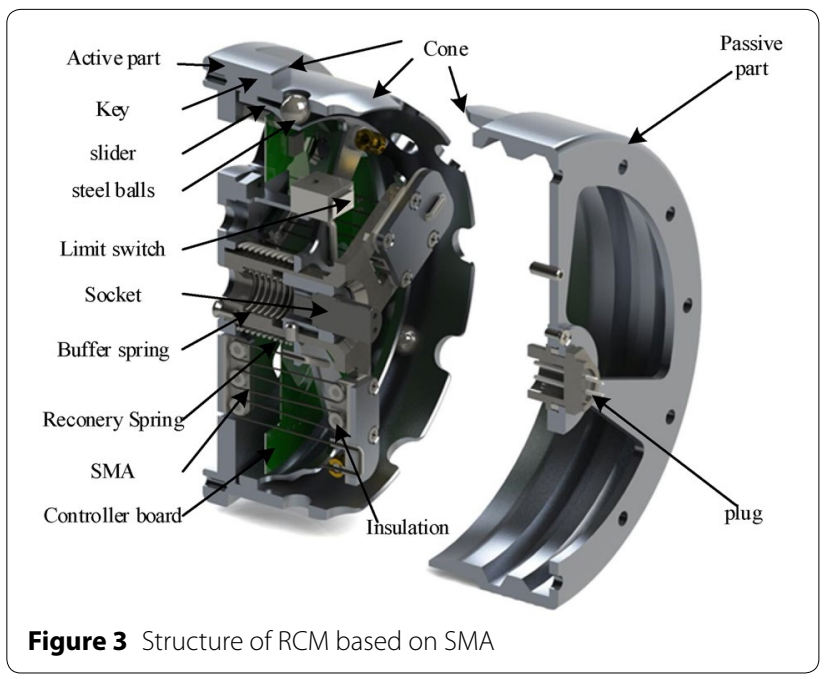




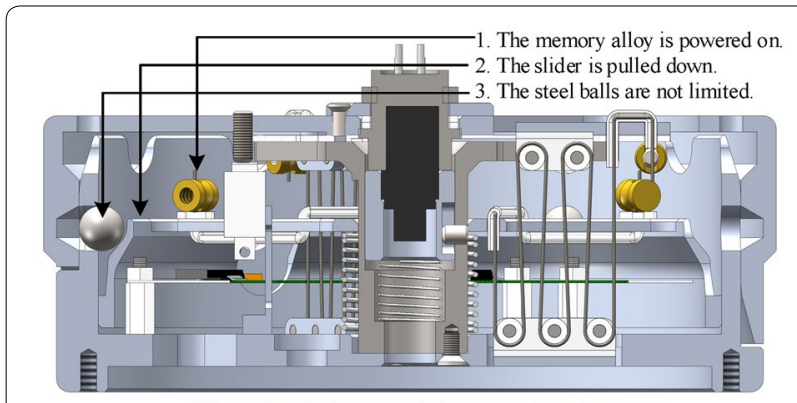

a The unlocked state of the RCM based on SMA

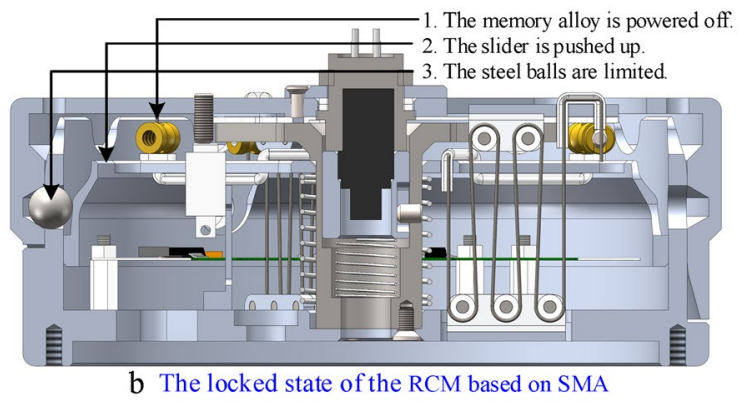

Figure 4 State of the RCM based on SMA

When the SMA is powered off, SMA returns to its original length. The slider is pushed to the position where the steel balls are limited by the cylindrical surface of the slider and the groove under the recovery spring. The $\mathrm{RCM}$ is in the locked state as shown in Figure 4(b). The steel balls and the key can undertake enough force and torque respectively. The SMA should be long enough to ensure the expanded length. Therefore, the s-shaped winding assembly is adopted.

The connection process of the active part and the passive part is as follows. When the SMA is powered on, the active part can begin to connect to the passive part. First, the active part moves to the initial position within the docking tolerance. Then, the axis of the active part is gradually aligned with the axis of the passive part under the guidance of the cone. The key of the active part is fitted into the keyway of the passive part under the guidance of the cone too. After that, the socket and the plug begin to connect under the buffer by the buffer spring. Finally, when the limit switch is triggered, the SMA is powered off. The connection process is completed.

\subsection{Mechanism Design}

\subsubsection{Tolerance Design}

In order to ensure that the connection process can be achieved successfully under the position and attitude errors, we designed large tolerance structures on the RCM as Figure 5. The taper surfaces are set where the active part and the passive part begin to connect. The pins and sockets of selected electrical connector also

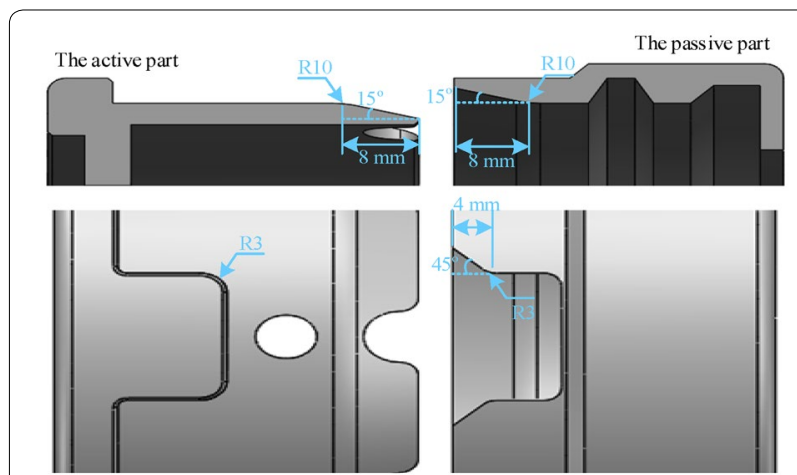

Figure 5 Tolerance designs of the RCM based on SMA

have a small taper. With these designs, the RCM based on SMA has a position tolerance of $\pm 8 \mathrm{~mm}$ and an angle tolerance of $\pm 7.5^{\circ}$.

\subsubsection{Locking Mechanism}

The steel balls restrict 5 degrees of freedom of the RCM except the freedom of rotation around the central axis. This degree of freedom is constrained by the key on the RCM. The ring groove of the passive part, the hole of the active part, and the conical surface of the slider restrict the movement of the steel balls together. The locking mechanism is shown in Figure 6. The design parameters are as follows.

The cone angle of the ring groove is $\alpha_{b}$. The steel ball radius is $r_{b a l l}$. The maximum width of the ring groove is $w_{b}$. The moving distance of the steel ball is $d_{b a l l}$. The cone angle of the first conical surface on the slide is $\beta_{s}$. The cone angle of the second conical surface on the slide is $\gamma_{s}$.

To produce a stable and effective locking force, it follows that,

$$
w_{b}>2 r_{b a l l} \cos \alpha_{b}
$$

According to Figure 6, the parameters have the following relationships:

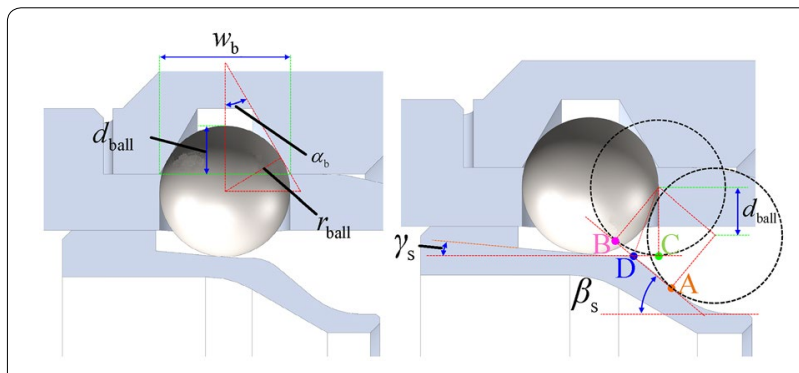

Figure 6 Tolerance designs of the RCM based on SMA 


$$
\begin{aligned}
& d_{\text {ball }}=r_{\text {ball }}-\left(\frac{r_{\text {ball }}}{\sin \left(\alpha_{b}\right)}-\frac{w_{b}}{2 \tan \left(\alpha_{b}\right)}\right), \\
& l_{\text {slider }}=\frac{d_{\text {ball }}}{\tan \left(\beta_{s}\right)}+r_{\text {ball }} \tan \left(\frac{\beta_{s}}{2}\right) .
\end{aligned}
$$

The depth of the ring groove should be greater than the movement of the steel balls. The clearance fit is designed between the hole and the steel ball. The steel ball can move in the hole with low friction. In order to prevent the steel ball from escaping from the hole, the cylindrical hole is convergent to arc-shaped hole with the smaller diameter than the diameter of the steel ball. In order to facilitate processing, the opposite side of the hole is punched. Three steel balls are chosen to lock the RCM.

\subsubsection{Slider}

Two states are generated as the slider moves along the central axis of the RCM. The position of the locking state is called the locking position. The position of the unlocking state is called the unlocking position. The slider design is shown in Figure 7.

The slider is designed with two conical surfaces corresponding to the locking position and the unlocking position respectively. To ensure full unlocking, the vertical distance between the locking position and the locked position $d_{\text {slider }}$ should be greater than the steel ball displacement $d_{\text {ball }}$. To ensure full locking, the conical surface is designed on the unlocking position. The steel ball is tightly contacted with the ring groove under the force of the conical surface. The reliability and

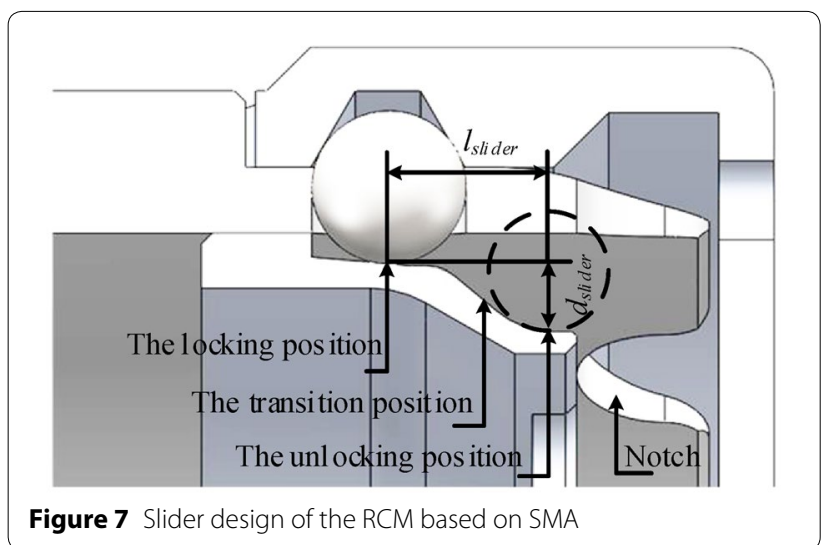

accuracy of the RCM are high. To facilitate the installation of steel ball and reduce mass, some notches are designed in the slider. The steel ball can be installed from the notch. The slider rotates a certain angle to make the steel ball fall into the hole. If the horizontal displacement of the slider $l_{\text {slider }}$ is short, it will not only help reduce the size of the RCM, but also reduce the design requirements of the driver. According to the above analysis, the parameters of the RCM are shown in Table 1. The calculated result is $l_{\text {slider }}=4.33 \mathrm{~mm}$. To ensure lock stability, the displacement of the slider $l_{\text {slider }}$ is chosen as $l_{\text {slider }}=6 \mathrm{~mm}$.

\subsubsection{SMA-spring Combination Driving Source}

The SMA and the recovery spring are used to pull and push the slider respectively. When the RCM is applied to the reconfigurable manipulator, it needs to undertake certain payloads. Suppose that the maximum payload along its axis is $500 \mathrm{~N}$. The analysis of the force on the ball is shown in Figure 8.

According to the force balance equations, we can get

$$
\begin{aligned}
& F_{f b}=\frac{F_{e x}}{n \cos \left(\alpha_{b}\right)}, \\
& F_{s b} \cos \left(\gamma_{s}\right)=F_{f b} \sin \left(\alpha_{b}\right),
\end{aligned}
$$

where $F_{f b}$ is the force acting on the ball by the passive part. $F_{e x}$ is the force acting on the RCM along the central axis. $F_{s b}$ is the force acting on the ball by the slider. $n$ is the number of the steel balls. In this paper, $n$ is equal to 3 .

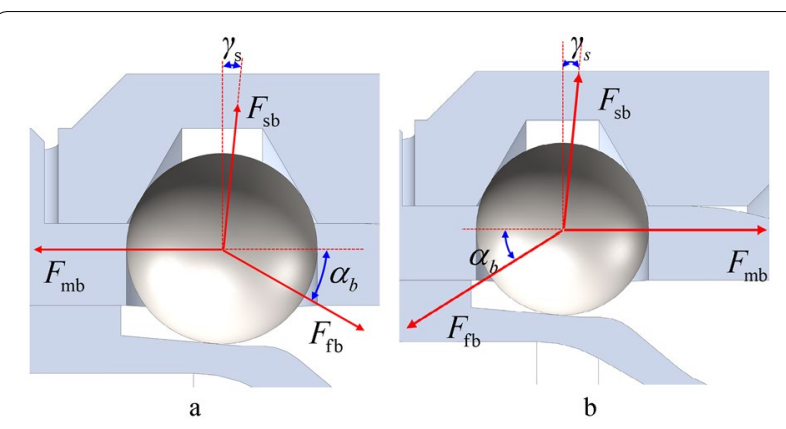

Figure 8 Analysis of the force on the ball: a) RCM is under the pulling force, $b$ ) RCM is under pressure

Table 1 Parameters of the RCM based on SMA

\begin{tabular}{lllllll}
\hline$r_{\text {ball }}(\mathrm{mm})$ & $\boldsymbol{w}_{\boldsymbol{b}}(\mathrm{mm})$ & $\boldsymbol{\alpha}_{\boldsymbol{b}}\left({ }^{\circ}\right)$ & $\boldsymbol{\beta}_{\boldsymbol{s}}\left({ }^{\circ}\right)$ & $\boldsymbol{\gamma}_{\boldsymbol{s}}\left({ }^{\circ}\right)$ & $\boldsymbol{d}_{\text {ball }}(\mathbf{m m})$ & $\boldsymbol{I}_{\text {slider }}(\mathrm{mm})$ \\
\hline 3.5 & 7 & 30 & 40 & 5 & 2.56 & 4.33 \\
\hline
\end{tabular}


For the slider, the resultant force by the pressure from the steel balls and the pushing force from the recovery spring are balanced. It follows that,

$$
F_{L}=n F_{b s} \sin \left(\gamma_{s}\right)
$$

where $F_{L}$ is the force acting on the slider by the recovery spring. $F_{b s}$ is the force acting on the slider by the balls.

Substituting Eqs. (4), (5) into Eq. (6), we can get

$$
F_{L}=F_{e x} \tan \left(\alpha_{b}\right) \sin \left(\gamma_{s}\right) .
$$

The force $F_{L}$ can be obtained with Eq. (7) and the given parameters. The calculated result is $F_{L}=25.16 \mathrm{~N}$. To ensure lock stability, the force $F_{L}$ is chosen as $F_{L}=30 \mathrm{~N}$.

According to the calculation of the horizontal displacement $l_{\text {slider }}$ and the pushing force $F_{L}$ by the recovery spring, the SMA-spring driving source needs to move $6 \mathrm{~mm}$ and withstands the payload of $30 \mathrm{~N}$.

The SMA-spring combination driving source has the advantages of light weight and simple control comparing with the motor, hydraulic and pneumatic drives. The SMA-spring combination driving source is shown in Figure 9.

In order to generate the resultant force along the central axis so that the resultant torque is zero, three SMA wires are evenly distributed around the central axis. The SMA wires adopt s-shaped installation to guarantee sufficient length. The phase transition temperature, rated current (A), and shrinkage ratio of the SMA have the greatest influence on its working performance. The parameters of the SMA are set in Table 2.

\subsubsection{Insulation}

The SMA wires need to be installed into the limited internal space of the RCM. Therefore, we adopt the $\mathrm{s}$-shape installation method. The memory alloy wire will contact with multiple parts, such as pins, screws, etc. These parts are usually made of metal which need to be insulated from the SMA wires. Using the insulation gaskets and polytetrafluoroethylene (PTFE) tubes, the insulation between the SMA wire and the other parts can be achieved. PTFE material has good insulation, flame resistance, and pressure resistance. It can work in low

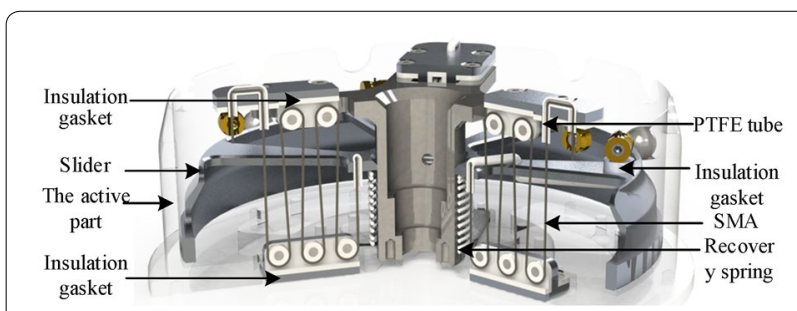

Figure 9 SMA-spring combination driving source
Table 2 Parameters of the Ni-Ti SMA

\begin{tabular}{lllll}
\hline $\begin{array}{l}\text { Diameter } \\
(\mathbf{m m})\end{array}$ & Length $(\mathbf{m m})$ & Voltage (V) & Current (A) & $\begin{array}{l}\text { Resistance } \\
(\boldsymbol{\Omega})\end{array}$ \\
\hline 0.5 & 250 & 2 & 1.5 & 1.67 \\
\hline
\end{tabular}

temperature and high temperature environment for a long time. It is also a good self-lubricating material. The insulation parts are shown in Figure 10.

\subsection{Control System Design}

The RCM does not use the motor as the driving source. It only needs to power a certain magnitude of current (A) to the SMA to control its states. The control system includes the power conversion module, the communication module, and the current on-off module. The control system uses the ARM processor as the controller, the CAN bus as the communication module, and the thyristor as the current on-off module. The bluetooth is used for easy debugging and remote control. A half-bridge circuit composed of the thyristor management chip and thermistors is used to supply power to the SMA wire. The magnitude of the current (A) is controlled by PWM waves. A Light Emitting Diode (LED) is installed on the control board to indicate the status of the RCM. If the LED is on, it means that the SMA is powered, and the RCM is under the unlocked state. The internal space of the RCM is small. The circuit board is designed according to the mechanical structure. The control board of the RCM based on the SMA and installation are shown in Figure 11.

The working process of RCM for changing the end effector is as follows. First, the central controller sends control instructions to the RCM. Then, the processor of the RCM generates a certain pulse width PWM wave. The SMA is powered, the LED is on and the RCM is under the unlocked state. The active part can be separated from the passive part. After, the active part of the RCM can be connected to the passive part of the other RCM. When the limit switch is triggered, the processor of the RCM

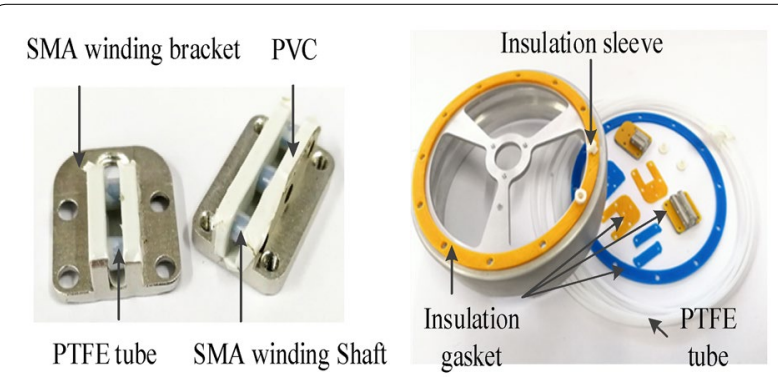

Figure 10 Insulation parts 


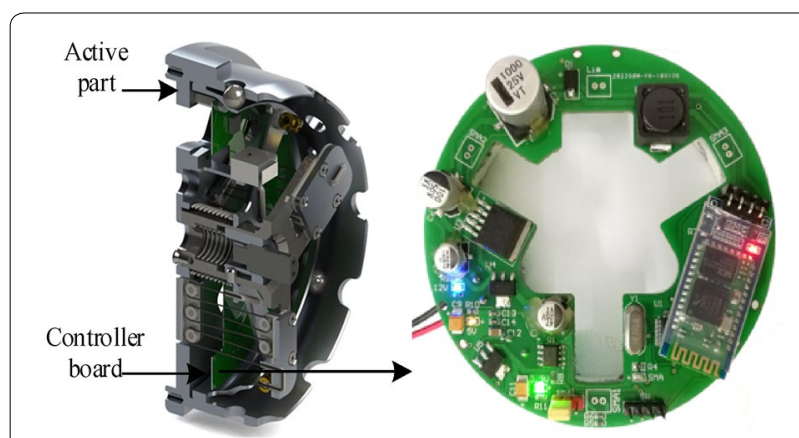

Figure 11 Control board and its installation of RCM based on SMA

stops the power supply. If the fault signal is generated during this period, the current task will be interrupted until the fault is eliminated.

\section{Electromagnetic-Driven Reconfigurable Mechanism}

\subsection{Structure Design}

\subsubsection{Overall Structure}

The overall structure of the electromagnetic RCM is shown in Figure 12. It includes the active part and the passive part. The active part undertakes most of the connecting works. It mainly consists of the electromagnetic coil as the driving source, the permanent magnet and key as the load-bearing parts, the cone as the tolerance, the socket and buffer as the electrical connections, the insulation as the leakage parts, and the controller board as the power management. The passive part cooperates with the active part, which is mainly composed of the armature, the plug, the cone, and the keyway. The key is set on the outer cylindrical surface, and the main tolerance is set on the inner cylindrical surface. When the cones of the active part and the passive part are successfully fitted, the plug and the socket begin to connect to realize electrical connection. Main material of the RCM is aluminum

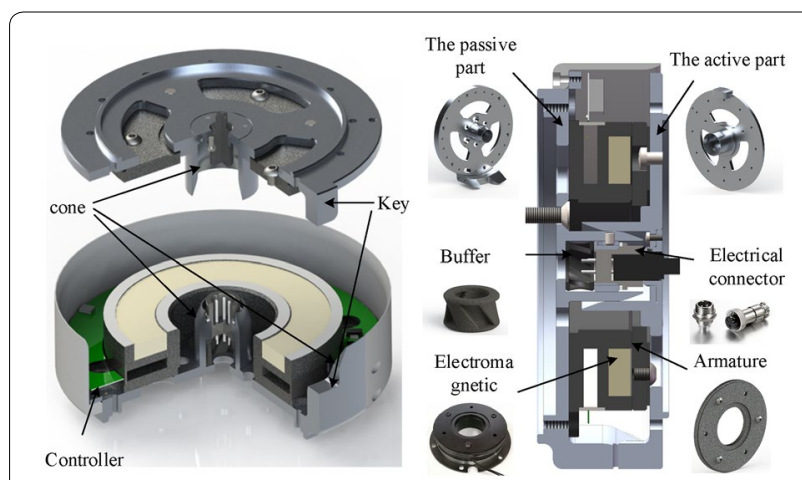

Figure 12 Structure of RCM based on electromagnetic alloy. In order to reduce weight, the unbearable parts are removed.

\subsubsection{Working Principle}

According to the power state of the electromagnetic coil, the reconfigurable interface can be divided into two states. When the electromagnetic coil is powered on, it generates a magnetic field that cancels out the magnetic field of the permanent magnet. There is no force on armature of the passive part by the active part. The RCM works in the unlocked state. The passive part can be separated from the active part. The unlocked state is shown in Figure 13(a).

When the electromagnetic coil is powered off, there is only the magnetic force on the armature by the permanent magnet. The RCM is under the locked state as shown in Figure 13(b). The amount of force can be designed. In this paper, we design the force being equal to $500 \mathrm{~N}$. The magnetic force is used to lock the active part and the passive part along the central axis. The key is used to lock the active part and the passive part around the central axis.

The connection process of the active part and the passive part is as follows. When the electromagnetic coil is powered on, the active part can begin to connect to the passive part. First, the active part moves to the initial position within the docking tolerance. Then, the axis of the active part is gradually aligned with the axis of the passive part under the guidance of the cone. The key of the active part is fitted into the keyway of the passive part under the guidance of the cone too. After that, the socket and the plug begin to connect under the buffer. Finally,

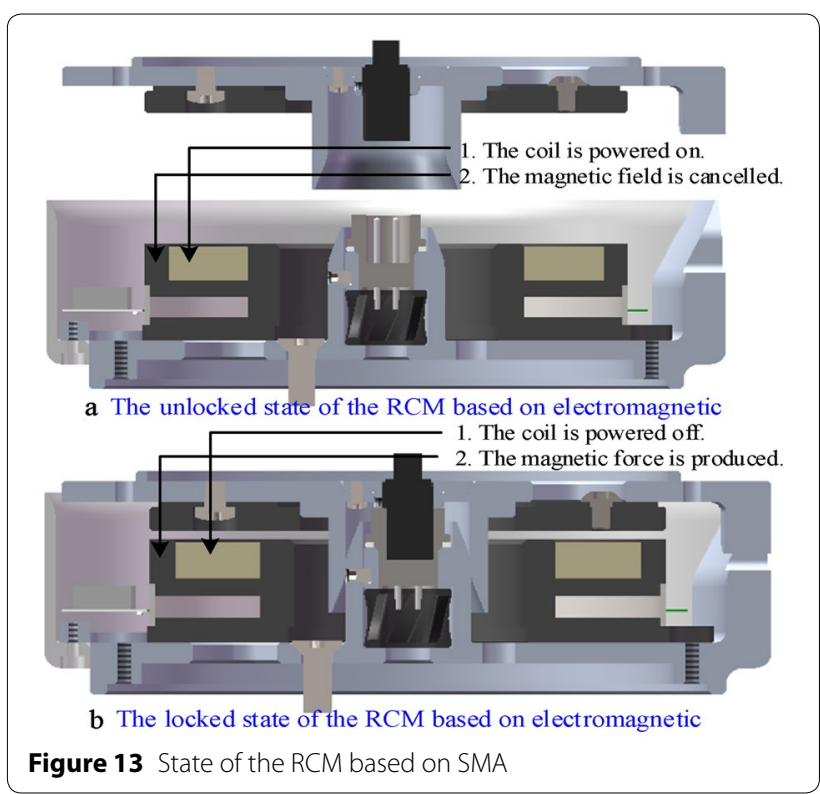


when the light is on, the electromagnetic coil is powered off. The connection process is completed.

\subsection{Mechanism Design}

\subsubsection{Tolerance Design}

In order to ensure that the connection process can be achieved successfully under the position and attitude errors, we designed large tolerance structures on the RCM. The taper surfaces are set where the active part and the passive part begin to adapt. The pins and sockets of selected electrical connector also have a small taper. With these designs, the electromagnetic RCM has a position tolerance of $\pm 7 \mathrm{~mm}$ and an angle tolerance of $\pm 10^{\circ}$. The tolerance designs are shown in Figure 14.

\subsubsection{Electromagnetic Driving Source}

The structure of the electromagnetic Driving Source is shown in Figure 15.

The electromagnetic coil are used to generate the magnetic field to cancel the magnetic field of the permanent magnet. When the RCM is applied to the reconfigurable manipulator, it needs to undertake certain payloads. Suppose that the maximum payload along its axis is $500 \mathrm{~N}$, the electromagnetic coil and the permanent magnet should generate the force of $500 \mathrm{~N}$ acting on the armature.

When no current passes through the coil, the permanent magnet generates the magnetic field to lock the armature. When the rated current is passed through the coil, the magnetic field produced by the coil will cancel the magnetic field of a permanent magnet. At this time, the armature can be separated from the magnet part.

In addition, the electromagnetic coil need to be installed into the limited internal space of the RCM. It needs to be insulated from the permanent magnet. The insulation of the electromagnetic RCM is simple. The

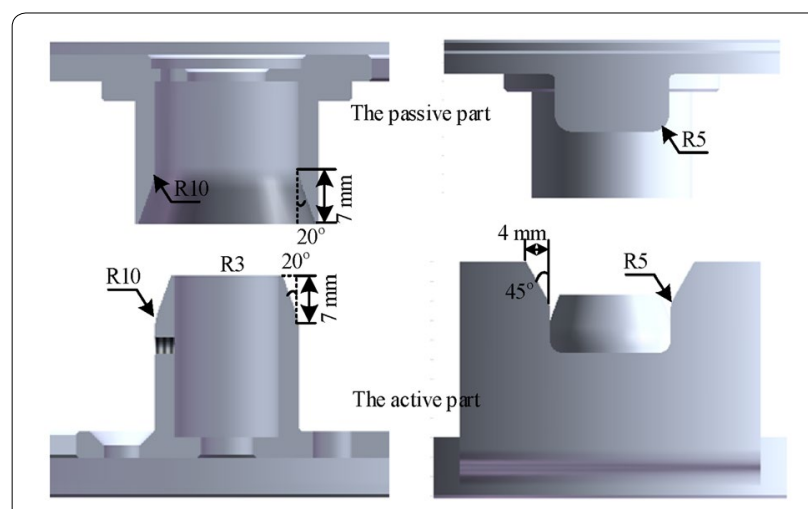

Figure 14 Tolerance designs of the RCM based on SMA

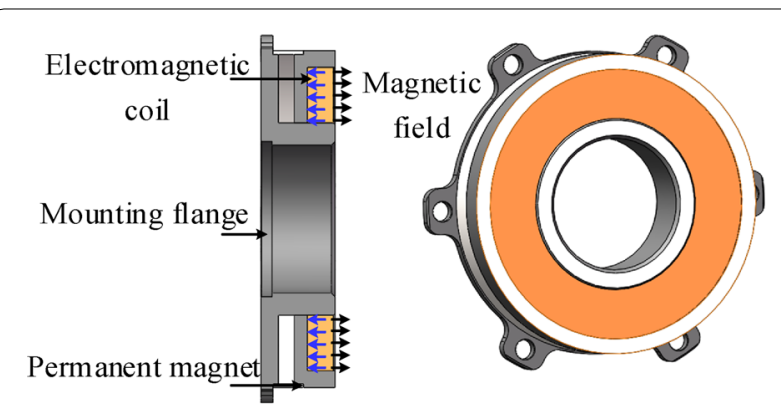

Figure 15 Structure of the electromagnetic driving source

insulated enameled wire is used for the coil and the insulation glue is used to fix the coil.

\subsection{Control System Design}

The electromagnetic RCM only needs to apply a certain amount of voltage to the electromagnetic coil to achieve the unlocking state. The control system includes the power conversion module, the communication module, and the current on-off module. The control system uses the ARM chip as the controller and the CAN bus to communicate with the manipulator. Bluetooth is equipped for easy debugging and remote control. The electromagnetic RCM also uses the half-bridge circuit to supply power to the coil. A Light Emitting Diode (LED) is installed on the control board to indicate the status of the RCM. If the LED is on, it means that the coil is powered, and the $\mathrm{RCM}$ is under the unlocked state. The internal space of the RCM is small. The circuit board is designed according to the mechanical structure. The control board of the RCM based on the electromagnetic and installation are shown in Figure 16.

The working process of RCM for changing the end effector is as follows. First, the central controller sends control instructions to the RCM. Then, the processor of the RCM generates a certain pulse width PWM wave. After the coil is powered, the RCM is under the locked state, and then the LED is on. The active part can be separated from the passive part. After, the active part of the RCM can be connected to the passive part of

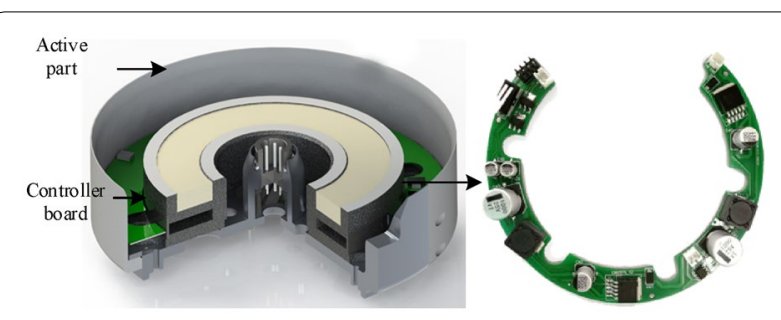

Figure 16 Structure of RCM based on electromagnetic 


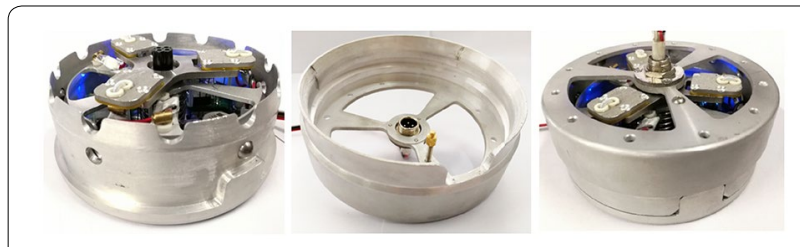

Figure 17 Prototype of the RCM based on SMA

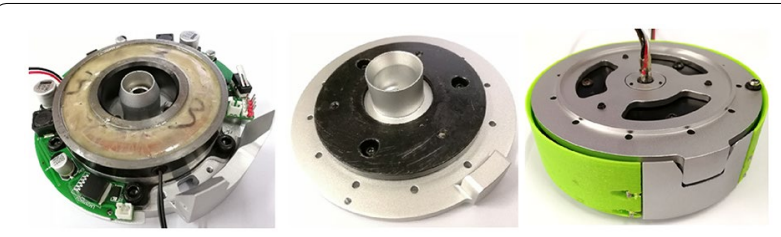

Figure 18 Prototype of the electromagnetic RCM

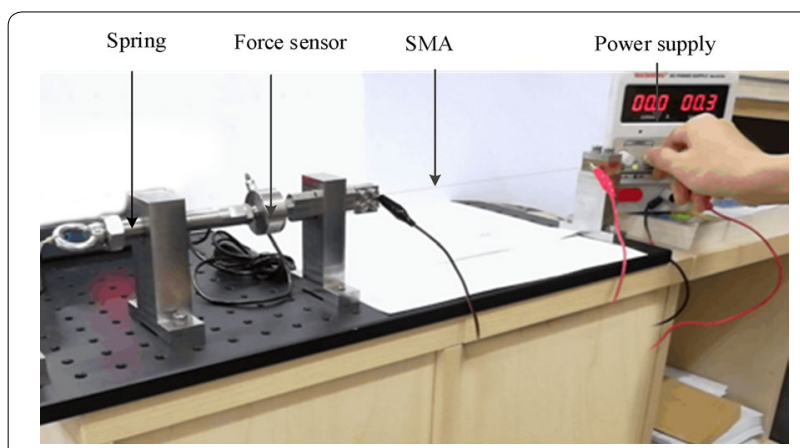

Figure 19 Performance testing platform of the SMA the other RCM. When the limit switch is triggered, the processor of the RCM stops the power supply. If the fault signal is generated during this period, the current task will be interrupted until the fault is eliminated.

\section{Experiments}

\subsection{Prototype}

In order to verify the performance of the two RCMs, we processed and assembled two prototypes. The RCM based on SMA is shown in Figure 17. The electromagnetic RCM is shown in Figure 18. We carried out the end effector connection experiment and the arm connection experiment.

\subsection{Performance Test of SMA-spring Combination Driver}

The SMA-spring combination driver is important to the RCM based on SMA. The performance test was performed on SMA wire with the diameter of $0.5 \mathrm{~mm}$ and a length of $200 \mathrm{~mm}$. The shrinkages of the SMA under different payloads at room temperature were examined. The payload was produced by the spring. Through adjusting the position of the screw to change the initial force. The experimental platform is shown in Figure 19. The force sensor is used to measure the force of the SMA. The experimental results are shown in Table 3.

From the data obtained from the experiment, it can be seen that the SMA has a rapid shrinkage when it is powered. The natural recovery time is slow when it loses power. The greater the current (A) applied, the shorter the time spent. The SMA-spring combination

Table 3 Experimental results of the SMA

\begin{tabular}{|c|c|c|c|c|c|c|}
\hline Initial force (N) & Final force (N) & Elongation (mm) & Voltage (V) & Current $(A)$ & Shrinking time (s) & $\begin{array}{l}\text { Recovery } \\
\text { time (s) }\end{array}$ \\
\hline 10.2 & 15.0 & 6.0 & 1.4 & 1.0 & 18 & 20 \\
\hline 10.2 & 15.3 & 6.0 & 1.6 & 1.2 & 14 & 18 \\
\hline 10.2 & 16.0 & 6.0 & 2.0 & 1.5 & 9 & 16 \\
\hline 15.3 & 22.0 & 7.0 & 1.3 & 1.0 & 20 & 20 \\
\hline 15.3 & 22.4 & 7.0 & 1.6 & 1.2 & 14 & 16 \\
\hline 15.3 & 23.0 & 7.0 & 2.0 & 1.5 & 8 & 12 \\
\hline 20.0 & 26.5 & 7.1 & 1.4 & 1.0 & 16 & 22 \\
\hline 20.0 & 27.0 & 7.0 & 1.6 & 1.2 & 12 & 18 \\
\hline 20.0 & 28.0 & 7.0 & 2.0 & 1.5 & 10 & 14 \\
\hline 25.0 & 35.1 & 7.0 & 1.4 & 1.0 & 14 & 20 \\
\hline 25.0 & 36.2 & 7.0 & 1.6 & 1.2 & 10 & 16 \\
\hline 25.0 & 38.8 & 7.0 & 2.0 & 1.5 & 7 & 12 \\
\hline
\end{tabular}


driver needs to meet a displacement of $6 \mathrm{~mm}$ and a locking force of $30 \mathrm{~N}$. Each SMA wire will undertake 10 $\mathrm{N}$ payload. The current (A) which we apply to the SMA is $1.5 \mathrm{~A}$. The spent time is $7 \mathrm{~s}$ when the SMA shrinks by $7 \mathrm{~mm}$.

\subsection{End Tool Connection}

We use a 7-DOF modular manipulator to carry out the end effector connecting experiments. The experimental system includes a 7-DOF modular manipulator and its control system, the vision system for initial positioning, a force sensor for measuring the force of the docking process, an end effector, and two RCMs.

The connection process using the RCM based on SMA is shown in Figure 20. The connection process of the electromagnetic RCM is shown in the Figure 21.

When the SMA or the electromagnetic coil is powered, the RCM is in the unlocking state. When the power is lost, the RCM is locked. While the mechanical structure is connected, the power and communication are also connected. The light is on indicating that the connection process is successfully completed. Based on the above experiments, we summarize the indicators of the two RCMs in Table 4. The RCM based on SMA has a lower weight and power, but more reconfigurable time. The electromagnetic RCM has more compact dimensions in axis direction. The RCM based on SMA is more suitable for robot self-reconfiguration, while the electromagnetic RCM is more suitable for the tool replacement.

\section{Conclusions}

Two types of RCMs including the RCM based on SMA and the electromagnetic RCM are developed for the reconfigurable manipulator in industrial area. Due to their good performances of light weight, high payload,

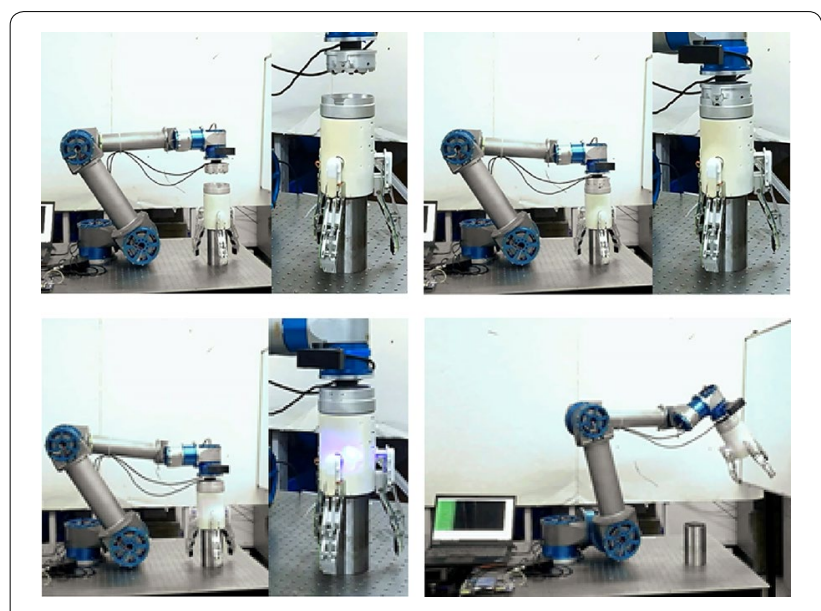

Figure 20 Connection process using the RCM based on SMA

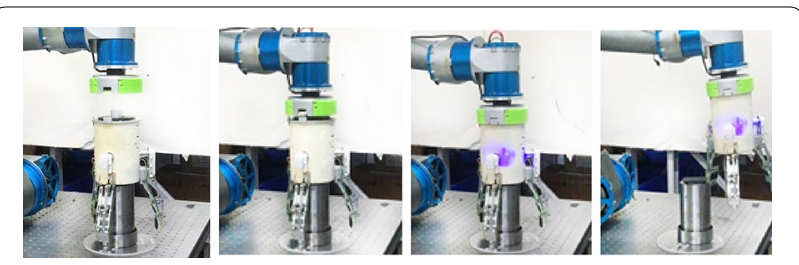

Figure 21 Connection process of the electromagnetic RCM

Table 4 The indicators of the two RCMs

\begin{tabular}{lll}
\hline Indicators & RCM (SMA) & RCM (electromagnetic) \\
\hline Mass (kg) & 0.15 & 0.32 \\
Payload (N) & 500 & 500 \\
Tolerance & $\pm 8 \mathrm{~mm}, \pm 7.5^{\circ}$ & $\pm 7 \mathrm{~mm}, \pm 10^{\circ}$ \\
Time $(\mathrm{s})$ & $\leq 10$ & $\leq 4$ \\
Force & $\leq 10 \mathrm{~N}, 4 \mathrm{~N} \cdot \mathrm{m}$ & $\leq 5 \mathrm{~N}, 2 \mathrm{~N} \cdot \mathrm{m}$ \\
Size & $\Phi 114 \times 42.95 \mathrm{~mm}$ & $\Phi 128 \times 37.6 \mathrm{~mm}$ \\
Power & $2 \mathrm{~V}, 1.5 \mathrm{~A}$ & $24 \mathrm{~V}, 0.8 \mathrm{~A}$ \\
\hline
\end{tabular}

high reliability and big tolerance, the RCMs has a great application prospect.

We developed the RCM based on SMA. It uses the SMA and recovery spring as the driving source. The steel balls and key are used as the load-bearing parts. Mechanical design, and the control system design are introduced in detail.

We developed the electromagnetic RCM. It uses the electromagnetic coil as the driving source. The permanent magnet and key are used as the load-bearing parts. Mechanical design, and the control system design are introduced in detail also. Compared with the RCM based on SMA, the electromagnetic RCM has simpler structure, but heavier mass and lower locking force.

Finally, we build two prototype and carry out a lot of experiments including the test of SMA-spring combination driver and end tool connection test. The results show that both two RCMs have good performances. In the future, we will verify the RCMs in self-reconfigurable manipulators. The changes of the joint, the link, the end effector, and the configuration will be deeply studied.

\section{Acknowledgements}

The authors sincerely thanks to Professor Bing Li of Harbin Institute of Technology for his critical discussion and reading during manuscript preparation.

\section{Authors' contributions}

XW proposed the design idea and supplied laboratory setup; $\mathrm{HL}$ wrote the manuscript; WX supplied concept design assisted with sampling and laboratory analyses. YH was in charge of the whole trial; LB helped perform the analysis with constructive discussions. All authors read and approved the final manuscript. 


\section{Authors' Information}

Wenfu Xu, born in 1979, is currently a professor at School of Mechanical Engineering and Automation, Harbin Institute of Technology, Shenzhen, China. He received his B.E. degree in 2001, and M.E. degree in 2003, respectively in Automation, Detection Technology and Its Automation Device, from Hefei University of Technology, China, and his Ph.D. degrees in Control Science and Engineering from Harbin Institute of Technology, Harbin, China, in 2007. His research interests include reconfigurable robots, bionic robots and space robotics.

Liang Han, born in 1993, is currently a PhD candidate at School of Mechanical Engineering and Automation, Harbin Institute of Technology, Shenzhen, China. He received his master degree from Harbin Institute of Technology, Shenzhen, China, in 2016. He received his bachelor degree from Hefei University of Technology, China, in 2014. His research interests include the reconfigurable interface, and human-robot interaction.

Xin Wang, born in 1992, is currently a research assistant at School of Mechanical Engineering and Automation, Harbin Institute of Technology, Shenzhen, China. He received his B.E. degree in Mechanical and Automotive Engineering from Hefei University of Technology, China, in 2016. He received his master degree from Harbin Institute of Technology, Shenzhen, China, in 2018. His research interests include the RCM, dexterous hands, and compliance control.

Han Yuan born in 1987, is currently an assistant professor at School of Mechanical Engineering and Automation, Harbin Institute of Technology, Shenzhen, China. He received his Ph.D. degree in Mechanical Engineering from Institute National des Sciences Appliquées de Rennes, Rennes, France, in 2015. He was a Research Scientist in CNRS UMR6602, Clermont-Ferrand, France from 2015 to 2016 and a Postdoctoral Fellow in the Chinese University of Hong Kong, Hong Kong, China, from 2016 to 2017. His research interests include robots, cable-driven manipulator and flexible actuators.

Bin Liang, born in 1968, is currently a professor at Department of Automation, Tsinghua University, China. He received his Ph.D degree in precision instrument and mechanology from Tsinghua University, China, in 1994. His research interests include space robotics, manipulators, and intelligent control.

\section{Funding}

Supported by National Key R\&D Program of China (Grant No. 2018YFB1304600), Guangdong Provincial Key Research and Development Program of China (Grant No. 2019B090915001), Guangdong Provincial Special Support Program of China (Grant No. 2017TX04X0071), and Basic Research Program of Shenzhen City (Grant No. JCYJ20180507183610564).

\section{Competing interests}

The authors declare no competing financial interests.

\section{Author Details}

${ }^{1}$ School of Mechanical Engineering and Automation, Harbin Institute of Technology, Shenzhen 518055, China. ${ }^{2}$ Department of Automation, Tsinghua University, Beijing 100084, China.

Received: 27 March 2020 Revised: 15 September 2020 Accepted: 14 October 2020

Published online: 28 October 2020

\section{References}

[1] M Bortolini, F G Galizia, C Mora. Reconfigurable manufacturing systems: Literature review and research trend. Journal of Manufacturing Systems, 2018, 49: 93-106.

[2] C J Paredis, H B Brown, P K Khosla. A rapidly deployable manipulator system. Robotics and Autonomous Systems, 1997, 21(3): 289-304.

[3] M Plooij, G Mathijssen, P Cherelle, et al. Lock your robot: A review of locking devices in robotics. IEEE Robotics \& Automation Magazine, 2015, 22(1): 106-117.
[4] A Valente. Reconfigurable industrial robots: A stochastic programming approach for designing and assembling robotic arms. Robotics and Computer-Integrated Manufacturing, 2016, 41: 115-126.

[5] J Mukundan, J F Martinez. MORSE: Multi-objective reconfigurable self-optimizing memory scheduler. IEEE International Symposium on HighPerformance Comp Architecture, New Orleans, LA, USA, 2012: 1-12.

[6] I Chen. Rapid response manufacturing through a rapidly reconfigurable robotic workcell. Robotics and Computer-Integrated Manufacturing, 2001, 17(3): 199-213.

[7] T Zhang, W Zhang, M M Gupta. An underactuated self-reconfigurable robot and the reconfiguration evolution. Mechanism and Machine Theory, 2018, 124: 248-258

[8] G Liu, Y Liu, A A Goldenberg. Design, analysis, and control of a springassisted modular and reconfigurable robot. IEEE/ASME Transactions on Mechatronics, 2010, 16(4): 695-706.

[9] TMatsumaru. Design and control of the modular robot system: TOMMS. IEEE International Conference on Robotics and Automation, Nagoya, Japan, 1995: 2125-2131.

[10] G Hirzinger, N Sporer, A Albu-Schaffer, et al. DLR's torque-controlled light weight robot III-are we reaching the technological limits now? IEEE International Conference on Robotics and Automation, Washington, DC, USA, 2002: 1710-1716.

[11] Z Bobovský, V Krys, J Babjak, et al. Connecting system for quick replacement of mechatronic SCHUNK power cube modules for mobile robotic systems. Applied Mechanics and Materrials, 2015, 772: 318-323.

[12] F Aghili, K Parsa. A reconfigurable robot with lockable cylindrical joints. IEEE Transactions on Robotics, 2009, 25(4): 785-797.

[13] M Yim, W Shen, B Salemi, et al. Modular self-reconfigurable robot systems [grand challenges of robotics]. IEEE Robotics \& Automation Magazine, 2007, 14(1): 43-52.

[14] T Kislassi, D Zarrouk. A minimally actuated reconfigurable continuous track robot. IEEE Robotics and Automation Letters, 2019, 3(2): 652-659.

[15] M E Karagozler, S C Goldstein, J R Reid. Stress-driven mems assembly + electrostatic forces $=1 \mathrm{~mm}$ diameter robot. IEEE/RSJ International Conference on Intelligent Robots and Systems, St. Louis, MO, USA, 2009: 2763-2769.

[16] Y Zhu, J Zhao, X Cui, et al. Design and implementation of ubot: A modular self-reconfigurable robot. IEEE International Conference on Mechatronics and Automation, Takamatsu, Japan, 2013: 1217-1222.

[17] S Kamon, N Bunathuek, P Laksanacharoen. Development of a reconfigurable spherical robot III. Research, Invention, and Innovation Congress, Bangkok, Thailand, 2019: 1-5.

[18] M Yim, D G Duff, K D Roufas. PolyBot: A modular reconfigurable robot. IEEE International Conference on Robotics and Automation, San Francisco, CA, USA, 2000: 514-520.

[19] M Yim, Y Zhang, K Roufas, et al. Connecting and disconnecting for chain self-reconfiguration with PolyBot. IEEE/ASME Transactions on Mechatronics, 2002, 7(4): 442-451.

[20] S Hauser, M Mutlu, P Léziart, et al. Roombots extended: Challenges in the next generation of self-reconfigurable modular robots and their application in adaptive and assistive furniture. Robotics and Autonomous Systems, 2020, 127: 103467.

[21] A Castano, A Behar, P M Will. The Conro modules for reconfigurable robots. IEEE/ASME Transactions on Mechatronics, 2002, 7(4): 403-409.

[22] V Prabakaran, M R Elara, T Pathmakumar, et al. Floor cleaning robot with reconfigurable mechanism. Automation in Construction, 2018, 91: 155-165.

[23] C H Belke, J Paik. Mori: A modular origami robot. IEEE/ASME Transactions on Mechatronics, 2017, 22(5): 2153-2164.

[24] F Mondada, G C Pettinaro, A Guignard, et al. SWARM-BOT: A new distributed robotic concept. Autonomous Robots, 2004, 17(2-3): 193-221.

[25] Z Luo, J Shang, G Wei, et al. A reconfigurable hybrid wheel-track mobile robot based on Watt II six-bar linkage. Mechanism and Machine Theory, 2018, 128: 16-32.

[26] D Zarrouk, L Yehezkel. Rising star: A highly reconfigurable sprawl tuned robot. IEEE Robotics and Automation Letters, 2018, 3(3): 1888-1895.

[27] S Murata, E Yoshida, A Kamimura, et al. M-TRAN: Self-reconfigurable modular robotic system. IEEE/ASME Transactions on Mechatronics, 2002, 7(4): 431-441. 
[28] H Kurokawa, A Kamimura, EYoshida, et al. M-TRAN II: Metamorphosis from a four-legged walker to a caterpillar. IEEE/RSJ International Conference on Intelligent Robots and Systems, Las Vegas, NV, USA, 2003: 2454-2459.

[29] H Kurokawa, KTomita, A Kamimura, et al. Distributed self-reconfiguration of M-TRAN III modular robotic system. The International Journal of Robotics Research, 2008, 27(3-4): 373-386.

[30] B Salemi, M Moll, W Shen. SUPERBOT: A deployable, multi-functional, and modular self-reconfigurable robotic system. IEEE/RSJ International
Conference on Intelligent Robots and Systems, Beijing, China, 2006: 3636-3641.

[31] X Cui, Y Zhu, X Zang, et al. CPG based locomotion control of pitch-yaw connecting modular self-reconfigurable robots. IEEE International Conference on Information and Automation, Harbin, China, 2010: 1410-1415.

[32] Y Zhu, X Wang, X Cui, et al. Research on locomotive evolution based on worm-shaped configuration of self-reconfigurable robot HitMSR II. Electrical Power Systems and Computers, 2011: 245-252.

\section{Submit your manuscript to a SpringerOpen ${ }^{\circ}$ journal and benefit from:}

- Convenient online submission

- Rigorous peer review

- Open access: articles freely available online

- High visibility within the field

- Retaining the copyright to your article

Submit your next manuscript at $\boldsymbol{\nabla}$ springeropen.com 